\title{
Evaluation of early skin or laryngeal reaction at oral food challenge
}

\author{
Toshinori Nakamura ${ }^{1}$, Yuki Okada ${ }^{1}$, Mayu Maeda $^{1}$, Taro Kamiya ${ }^{1}$, and Takanori Imai ${ }^{1}$ \\ ${ }^{1}$ Showa University School of Medicine
}

January 29, 2021

\begin{abstract}
Background: An oral food challenge (OFC) is required for diagnosing food allergies; however, uncertain reactions can impair the determination of when to stop the test. We aimed to determine the associations between immediately occurring mild allergic skin signs/laryngeal symptoms and positive OFC results. Methods: We retrospectively included children (aged 6 months to 15 years) who underwent open OFC for hen's egg (HE), cow's milk (CM), or wheat at a single centre between May 2012 and March 2020. Participants with mild skin signs or laryngeal symptoms at OFC initiation were classified as "skin" or "laryngeal" cases, respectively. Using logistic regression, the risk of positive OFC results, in a skin or laryngeal case, was assessed using univariate and multivariate analyses. Age, sex, total target dose, and serum levels of total and food-specific immunoglobulin E were used as covariates in prediction models. Results: In total, 2954, 1126, and 850 tests for HE, CM, and wheat, respectively, were included and comprised $115(4 \%)$ and $25(0.9 \%), 92(9 \%)$ and $24(2 \%)$, and $7(1.3 \%)$ and $0(0 \%)$ skin and laryngeal cases, respectively. Children with reactions to both HE and CM had a higher risk of a positive OFC than controls (odds ratio [95\% confidence interval]: 4.6 [3.3-6.4], 2.9 [2.0-4.1] and 6.5 [3.0-10.9], 4.9 [2.2-10.9], respectively). Areas under the curves of prediction models ranged from 0.61 to 0.71 . Conclusions: Uncertain reactions immediately after test initiation could not robustly predict $\mathrm{OFC}$ results, indicating the OFC could be continued under careful observation.
\end{abstract}

Conflicts of interest:The authors declare that they have no conflicts of interests.

Financial support: This study received no specific grant from any funding agency.

Abstract

Background: An oral food challenge (OFC) is required for diagnosing food allergies; however, uncertain reactions can impair the determination of when to stop the test. We aimed to determine the associations between immediately occurring mild allergic skin signs/laryngeal symptoms and positive OFC results.

Methods: We retrospectively included children (aged 6 months to 15 years) who underwent open OFC for hen's egg (HE), cow's milk (CM), or wheat at a single centre between May 2012 and March 2020. Participants with mild skin signs or laryngeal symptoms at OFC initiation were classified as "skin" or "laryngeal" cases, respectively. Using logistic regression, the risk of positive OFC results, in a skin or laryngeal case, was assessed using univariate and multivariate analyses. Age, sex, total target dose, and serum levels of total and food-specific immunoglobulin E were used as covariates in prediction models.

Results: In total, 2954, 1126, and 850 tests for HE, CM, and wheat, respectively, were included and comprised $115(4 \%)$ and $25(0.9 \%), 92(9 \%)$ and $24(2 \%)$, and $7(1.3 \%)$ and $0(0 \%)$ skin and laryngeal cases, respectively. Children with reactions to both HE and CM had a higher risk of a positive OFC than controls (odds ratio [95\% confidence interval]: 4.6 [3.3-6.4], 2.9 [2.0-4.1] and 6.5 [3.0-10.9], 4.9 [2.2-10.9], respectively). Areas under the curves of prediction models ranged from 0.61 to 0.71 .

Conclusions: Uncertain reactions immediately after test initiation could not robustly predict OFC results, indicating the OFC could be continued under careful observation. 


\section{Key Words}

Anaphylaxis, food hypersensitivity, immunoglobulin E, larynx, logistic models

\section{Introduction}

An oral food challenge (OFC) is the gold standard for diagnosing food allergy. ${ }^{1}$ In immunoglobulin E (IgE)mediated food allergy, immunological tests for allergen-specific $\operatorname{IgE}$ or skin prick tests have predicted positive OFC results only for a few foods. ${ }^{2,}{ }^{3}$ Unnecessary dietary elimination of certain foods can lead to stunted growth and lower the quality of life. ${ }^{4,5}$ Therefore, an OFC should be conducted for patients suspected to have a food allergy, despite the associated risk of an allergic reaction. ${ }^{1}$ In an open OFC, the patient is aware of the food being tested. ${ }^{1}$ An OFC is considered positive if obvious allergy-related signs emerge. ${ }^{6}$ However, due to concerns about more severe reactions, examiners are likely to stop the test when uncertain reactions, such as mild localised skin signs or mild laryngeal symptoms, occur at test initiation, which results in an nonconclusive outcome. ${ }^{7}$ The Japanese guidelines recommend confirmation of the reproducibility of the reactions at home for non-conclusive results. ${ }^{6}$ European and US guidelines recommend a blinded OFC. ${ }^{8}, 9$ However, a blinded OFC tends to be expensive and time-consuming, which makes it inaccessible at many institutions. ${ }^{2}, 10$ Therefore, at our institution, when obvious signs occur, OFCs are either stopped or continued under careful monitoring.

Determining when to stop OFCs should be balanced between acquiring conclusive results and considering patient safety. However, it is unclear how the early reactions can be associated with OFC results, and this lack of knowledge makes it difficult to decide when to explicitly stop an OFC. Thus, we aimed to assess the risk of having a positive OFC result and further severe reactions among the patients having the early skin and laryngeal reactions, determining if the OFC should be stopped at the timing of the reactions emerged.

\section{Methods}

Study design, setting, and participants

In this retrospective study, we analysed the data of patients aged 6 months to 15 years, who underwent an open OFC for hen's egg, cow's milk, or wheat between May 2012 and March 2020 at the Showa University Hospital, a tertiary medical centre in Tokyo. The Medical Ethics Committee of Showa University approved this study, which adhered to the guidelines of the Declaration of Helsinki. Informed consent was obtained using the opt-out method.

The participants had undergone open OFCs for the diagnosis of suspected IgE-mediated food allergy or for confirmation of outgrowing a food allergy. We excluded subjects based on the following criteria: 1) If OFCs were conducted with the aim of oral immunotherapies, or for diagnosing non-IgE-mediated allergy or exercise-induced food allergy; 2) If the subject developed signs or symptoms that did not meet case definitions within 5 minutes from the initial dosing of OFC; and 3) If subjects had an inconclusive OFC result.

\section{Protocol for $O F C$}

Eight trained physicians conducted all the OFCs according to the Japanese guidelines, ${ }^{6}$ which recommend conducting tests with three different total target doses: low, medium, and full, with a stepwise increase (Table E1). ${ }^{6}$ A pre-test assessment of the risk of anaphylaxis and allergic reactions for each patient was undertaken at our clinic. Patients with high levels of specific IgE and previous allergic reactions with lowdose foods were considered to be high-risk patients and underwent OFCs starting from the low dose. ${ }^{6,11}$ The remaining patients underwent the OFC starting from the medium dose. The challenge foods, including cooked hen's eggs, pasteurised milk, and Udon (Japanese wheat noodle), were prepared at patients' home and brought to our clinic. The challenge food could be mixed with other foods to avoid children refusing to consume the food. The total challenge dose was administered as a single dose or as two divided doses. Examiners kept monitoring any allergic signs or symptoms for at least 120 minutes after the test-start.

Allergic reactions and treatments 
The allergic reactions observed during an open OFC were referred to as signs and symptoms in this study based on objective and subjective criteria, respectively. Participants with severe multiorgan signs or with hypotension were diagnosed with anaphylaxis. ${ }^{6}, 12$ Adrenaline injection was administered to participants with anaphylaxis or severely compromised respiratory system. ${ }^{6,12}$ Uncertain reactions were defined as mild signs or symptoms that resolved spontaneously and fulfilled the following criteria: skin rashes, erythema, and up to three small wheals limited to a single body part (e.g., left-arm alone), mild nasopharyngeal or pharyngeal discomfort, mild abdominal pain, and an isolated cough. ${ }^{13}$ Participants with resolved uncertain reactions were instructed to subsequently consume a divided dose.

\section{Interpretation of $O F C$}

Results of tests were recorded as "positive," "negative," or "non-conclusive." ${ }^{6}$ A test was stopped and diagnosed as "positive" when the participant had obvious systemic signs. ${ }^{6,13}$ Participants with uncertain reactions who consumed the total target dose without further reactions were encouraged to consume the same amount of challenge foods several times at home until the next clinic visit (home challenge), which was generally 1-2 months after a test. Negative OFCs were those wherein participants had no signs or symptoms at the challenge and those with uncertain reactions but provoked no reactions at the home challenge. ${ }^{6,13}$ "nonconclusive" OFCs included the following participants: 1) patients without signs or symptoms who refused to eat the challenge food; 2) patients with uncertain reactions, failing to take the total target dose at a test; and 3) patients with uncertain reactions but did not undergo the home challenge.

\section{Definition for cases and control}

Children who developed the following skin signs or laryngeal symptoms within 5 minutes of the initial dosing were classified as early skin or laryngeal cases, respectively: skin rashes, erythema, and up to three small wheals limited to a single body part; and cases where the parents or patients reported mild nasopharyngeal or pharyngeal discomfort. The control group involved patients who did not develop signs or symptoms within 5 minutes of the initial dosing.

\section{Primary outcome}

The primary outcome was the risk of having a positive OFC result among the patients assigned to the skin and laryngeal groups.

\section{Current allergic complications}

Prior to a test, a physician ascertained whether a patient had current symptoms for atopic dermatitis, asthma, and allergic rhinitis.

\section{Elimination reasons}

Elimination reasons were confirmed by physicians at each OFC, which were categorised to "allergic tests" or "objective allergic reactions." "Objective allergic reactions" involved patients who eliminated the causative food because of allergic signs that were previously provoked on consumption. "Allergic tests" involved patients without allergic reactions but sensitization to the food.

\section{Laboratory studies}

Serum samples were obtained before the OFC and examined for the levels of total $\operatorname{IgE}$ ( $\operatorname{IgE}$ ) and food-specific $\operatorname{IgE}(\mathrm{sIgE})$ antibodies against egg-white or cow's milk using ImmunoCAP (Phadia, Uppsala, Sweden). IgE values $<0.1$ and $>100 \mathrm{kU}_{\mathrm{A}} / \mathrm{L}$ were coded as 0.1 and $101 \mathrm{kU} \mathrm{A} / \mathrm{L}$, respectively.

\section{Statistical analyses}

Patient characteristics, incidence of positive results, and use of adrenaline injections were compared between cases and controls. The Mann-Whitney $U$ and chi-square tests were used to compare continuous and categorical variables, respectively. Univariate and multivariate logistic regression analyses were performed to assess the magnitude of association between early skin or laryngeal cases and positive OFC results. Based 
on previous studies, ${ }^{9}$ we included age, sex, elimination reasons, and serum levels of total, food-specific $\mathrm{IgE}$, and total target dose as covariates. Total and specific IgE raw values were log-transformed $[\log (\mathrm{x}+1)]$ prior to analysis. We constructed prediction models for positive OFC results using logistic regression, accounting for the same covariates as in other multivariate analyses. The models' predictive performance was evaluated using a receiver operating characteristic curve analysis. P-values $<0.05$ were considered statistically significant. All analyses were performed in STATA version 14.0 (Stata Corp., College Station, TX, USA). Numerical values were reported as a median (interquartile range).

\section{Results}

Of the 6047 tests conducted in the study period, 4930 tests were included (Figure 1); 2954 (60\%), 1126 $(23 \%)$, and $850(17 \%)$ tests were for hen's egg, cow's milk, and wheat allergy, respectively. Cases of skin and laryngeal reactions involved $114(4 \%)$ and $25(0.9 \%), 91(9 \%)$ and $24(2 \%)$, and $7(0.9 \%)$ and $0(0 \%)$ trials with hen's egg, cow's milk, and wheat OFCs, respectively. Given the small number of cases associated with wheat, further analysis was restricted to cases with hen's egg and cow's milk allergies.

Table 1 shows the patients' demographic and clinical characteristics; the median age was 31 (20-52) months; and 2999 [61\%] subjects had eliminated foods for objective allergic reactions. Early skin and laryngeal reactions were observed within a median period of $2(0-4)$ and $3(1-4)$ minutes, respectively, after OFC initiation.

\section{Early skin signs}

Children with skin signs in hen's egg OFC were significantly older $(46$ [28-69] vs. 30 [20-51], $p<0.001)$ and had a higher prevalence of asthma than those in the control group (25 [22\%] vs. 386 [14\%], $p=0.01$; Table E2). In cow's milk OFCs, case and control participants did not significantly differ in age and proportions of concomitant allergic diseases. In both hen's-egg and cow's-milk OFCs, the proportion of positive results was significantly higher among cases than among controls (hen's egg: 77 [67\%] vs. 647 [23\%], $p<0.001$; cow's milk: $55[60 \%]$ vs. $282[28 \%], p<0.001)$. The frequency of adrenaline injection was significantly higher among cases than among controls (hen's egg: 5 [4\%] vs. 35 [1\%], $p=0.005$; cow's milk: 5 [5\%] vs. 17 [2\%], $p=0.01$; Table 2).

\section{Early laryngeal symptoms}

In both hen's-egg and cow's-milk OFCs, children with laryngeal symptoms were older than those in the control group (hen's egg: 84 [53-114] vs. 30 [20-51] months, $p<0.001$; cow's milk: 71 [52-77] vs. 34 [21-57], $p<0.001$; Table E3). Compared with controls, children in the cases had significantly higher levels of total IgE (hen's egg: 808 [397-1891] vs. 252 [86-710] kU $/ \mathrm{L}, p<0.001$; cow's milk: 742 [378-1278] vs. 272 [107-656] $\mathrm{IU} / \mathrm{mL}, p<0.001$ ) and specific IgE (hen's egg: 25.0 [8.6-83.4] vs. $13.7[6.0-32.5] \mathrm{kU}_{\mathrm{A}} / \mathrm{L}, p=0.02$; cow's milk: $12.4[6.6-29.6]$ vs. $\left.6.3[2.5-14.4] \mathrm{kU}_{\mathrm{A}} / \mathrm{L}, p=0.002\right)$. The rate of positive results was significantly higher among cases than among controls only in hen's-egg OFCs (hen's egg: 16 [64\%] vs. 647 [23\%], $p<0.001$; cow's milk: $11[45 \%]$ vs. $282[28 \%], p=0.06$; Table 2).

\section{Primary outcome}

In both hen's-egg and cow's-milk OFCs, multivariate analysis revealed that early skin signs were associated with an increased risk of a positive result (odds ratio $[\mathrm{OR}]=5.1,95 \%$ confidence interval $[\mathrm{CI}]: 3.8-7.0$, and $\mathrm{OR}=3.3,95 \%$ CI: 2.0-5.3, respectively). Laryngeal symptoms increased the risk of a positive result only among hen's-egg OFCs (hen's egg: OR=5.2, 95\% CI: 2.2-12.2; cow's milk: OR=1.5, 95\% CI: 0.6-4.0; Table $3)$.

\section{Performance of prediction models}

The area under the curve of the four prediction models for positive OFC results ranged from 0.64 to 0.68 , indicating low predictive accuracy (Figure 2). Positive predictive values of the models ranged from $48 \%$ to $67 \%$ (Table E4). 


\section{Discussion}

This study revealed that mild skin signs or laryngeal symptoms occurring within 5 minutes of open OFC initiation were associated with a 7-fold higher risk of a positive test result compared to the lack of an immediate reaction. However, predictive models based on the immediate reactions were not robust. Therefore, we concluded that OFC among children with these reactions could be continued under careful observation.

Previous reports for standardising OFC have proposed various timepoints for the termination an OFC, which are inconsistent. ${ }^{1,6,14,15}$ For example, a consensus on OFC between the US and European countries recommended that an OFC should stop when any objective symptoms occur, ${ }^{1}$ whereas the Japanese guidelines for FA recommended that an OFC should be interpreted as positive if obvious symptoms are induced. ${ }^{6}$ The lack of knowledge with regard to the association between the immediate reactions and the risks of further allergic reactions during an OFC has led to this inconsistency, thereby hindering the standardization of OFC procedures. This study's findings indicate that both mild skin signs and laryngeal symptoms might increase the risk of intolerance among either hen's-egg or cow's-milk OFCs. Continuation of an OFC after the emergence of immediate allergic reactions should be determined by the associated risk of severe reactions, such as anaphylaxis. In this study, the incidence of anaphylaxis among skin and laryngeal cases was consistent with those that were previously reported, that severe allergic reactions may occur in 3-28\% of OFCs. ${ }^{16-18}$ Approximately $8 \%$ of the children with laryngeal reactions to hen's egg progressed to anaphylaxis on continuing the OFC; therefore, these children should be considered at high risk of severe reactions. However, the level of anaphylactic risk and the poor robustness of the prediction models may indicate continuance of the OFCs among skin and laryngeal cases with the condition that well-trained medical staff prepared to handle an emergency closely monitor the test.

The strength of this study is that all OFCs were conducted at a single centre. A multi-centre study on OFCs has shown low agreement rates for the interpretation of OFC results among participating centres. ${ }^{19}$ This study involved only eight trained physicians who supervised all OFCs, allowing a minimization of observer bias and heterogeneity in OFC interpretation.

This study has several limitations. First, the OFC results were not determined using a double-blind, placebocontrolled food challenge. We eliminated tests with non-conclusive results because of the uncertainty. A psychological burden to eat previously eliminated foods may have hindered eating the food at home, ${ }^{20}$ resulting in a lower rate of tolerance in this study. Future studies should employ a blinded OFC. Second, the method of preparation of challenge foods may have influenced the incidence of skin and laryngeal reactions. In the cases where the patient refused the challenge food, the parents were allowed to conceal the challenge food in another food to reduce the psychological burden of the OFC. ${ }^{21}$ The incidence of immediate reactions might have been higher if the challenge foods had been provided without any vehicles. However, this limitation does not significantly affect the generalizability of the findings, as open OFCs using processed foods are common in the clinical setting. Third, this study's findings may be inapplicable to other OFCs because the risk of positive results among the children in the case group may vary with different challenge foods. The prevalence of the cases differed between hen's egg, cow's milk, and wheat. Moreover, the magnitude of a risk of a positive OFC differed considerably between cases related to hen's-egg and cow's-milk OFCs. These findings suggest that the risk of positive test results should be evaluated for each challenge food.

In summary, to prevent inconclusive results in open OFCs, physicians should balance the benefits of acquiring definitive diagnoses with the risk of triggering severe allergic reactions. ${ }^{7}$ Our findings indicate that it may be acceptable to temporarily stop an OFC when immediate skin or laryngeal reactions occur and then resume it under careful supervision. This approach may reduce the need for a blinded OFC in the clinical setting and improve the quality of life of patients by preventing unnecessary food elimination. Future studies should validate the risk of continuing an OFC among children with immediate reactions using a double-blind, placebo-controlled food challenge.

\section{Acknowledgements}

We thank the children who enrolled in this study and their parents. This study received no specific grant 
from any funding agency.

\section{Impact statement}

There is no consensus on whether an open oral food challenge should stop when mild reactions occur at the start and examiners tend to continue the challenge based on clinical experience. This study shows that early mild skin signs or laryngeal symptom during open oral food challenge increase the risk of a positive outcome but are not decisive enough to stop the test. The findings clarify the risks and benefits of continuing the test. allowing physicians and patients to have a better discussion of when to stop the test.

\section{References}

1. Nowak-Wegrzyn A, Assa'ad AH, Bahna SL, Bock SA, Sicherer SH, Teuber SS, et al. Work Group report: oral food challenge testing. J Allergy Clin Immunol. 2009;123(6 Suppl):S365-S83.

2. Panel NI-SE, Boyce JA, Assa'ad A, Burks AW, Jones SM, Sampson HA, et al. Guidelines for the diagnosis and management of food allergy in the United States: report of the NIAID-sponsored expert panel. J Allergy Clin Immunol. 2010;126(6 Suppl):S1-S58.

3. Soares-Weiser K, Takwoingi Y, Panesar SS, Muraro A, Werfel T, Hoffmann-Sommergruber K, et al. The diagnosis of food allergy: a systematic review and meta-analysis. Allergy. 2014;69(1):76-86.

4. Sicherer SH, Noone SA, Munoz-Furlong A. The impact of childhood food allergy on quality of life. Ann Allergy Asthma Immunol. 2001;87(6):461-464.

5. Kansen HM, Le TM, Meijer Y, Flokstra-de Blok BMJ, Welsing PMJ, van der Ent CK, et al. The impact of oral food challenges for food allergy on quality of life: A systematic review. Pediatr Allergy Immunol. 2018;29(5):527-537.

6. Ebisawa M, Ito K, Fujisawa T, Committee for Japanese Pediatric Guideline for Food Allergy TJSoPA, Clinical Immunology TJSoA. Japanese guidelines for food allergy 2017. Allergol Int. 2017;66(2):248-264.

7. Niggemann B. When is an oral food challenge positive? Allergy. 2010;65(1):2-6.

8. Sampson HA, Gerth van Wijk R, Bindslev-Jensen C, Sicherer S, Teuber SS, Burks AW, et al. Standardizing double-blind, placebo-controlled oral food challenges: American Academy of Allergy, Asthma \& Immunology-European Academy of Allergy and Clinical Immunology PRACTALL consensus report. J Allergy Clin Immunol. 2012;130(6):1260-1274.

9. Muraro A, Werfel T, Hoffmann-Sommergruber K, Roberts G, Beyer K, Bindslev-Jensen C, et al. EAACI food allergy and anaphylaxis guidelines: diagnosis and management of food allergy. Allergy. 2014;69(8):10081025 .

10. Lieberman JA, Cox AL, Vitale M, Sampson HA. Outcomes of office-based, open food challenges in the management of food allergy. J Allergy Clin Immunol. 2011;128(5):1120-1122.

11. Yanagida N, Okada Y, Sato S, Ebisawa M. New approach for food allergy management using low-dose oral food challenges and low-dose oral immunotherapies. Allergol Int. 2016;65(2):135-140.

12. Simons FE, Ardusso LR, Bilo MB, El-Gamal YM, Ledford DK, Ring J, et al. World allergy organization guidelines for the assessment and management of anaphylaxis. World Allergy Organ J. 2011;4(2):13-37.

13. Miura T, Yanagida N, Sato S, Ogura K, Ebisawa M. Follow-up of patients with uncertain symptoms during an oral food challenge is useful for diagnosis. Pediatr Allergy Immunol. 2018;29(1):66-71.

14. Bird JA, Leonard S, Groetch M, Assa'ad A, Cianferoni A, Clark A, et al. Conducting an Oral Food Challenge: An Update to the 2009 Adverse Reactions to Foods Committee Work Group Report. J Allergy Clin Immunol Pract. 2020;8(1):75-90 e17. 
15. Bird JA, Groetch M, Allen KJ, Bock SA, Leonard S, Nowak-Wegrzyn AH, et al. Conducting an Oral Food Challenge to Peanut in an Infant. The Journal of Allergy and Clin Immunol Pract. 2017;5(2):301-11.e1.

16. Yanagida N, Minoura T, Kitaoka S, Ebisawa M. A three-level stepwise oral food challenge for egg, milk, and wheat allergy. J Allergy Clin Immunol Pract. 2018;6(2):658-660 e10.

17. Mankad VS, Williams LW, Lee LA, LaBelle GS, Anstrom KJ, Burks AW. Safety of open food challenges in the office setting. Ann Allergy Asthma Immunol. 2008;100(5):469-474.

18. Perry TT, Matsui EC, Conover-Walker MK, Wood RA. Risk of oral food challenges. J Allergy Clin Immunol. 2004;114(5):1164-1168.

19. Grabenhenrich LB, Reich A, Bellach J, Trendelenburg V, Sprikkelman AB, Roberts G, et al. A new framework for the documentation and interpretation of oral food challenges in population-based and clinical research. Allergy. 2017;72(3):453-461.

20. Herbert LJ, Dahlquist LM, Bollinger ME. Maternal intolerance of uncertainty, anxiety, and adherence with food challenge referrals. J Health Psychol. 2013;18(9):1209-1219.

21. Yanagida N, Sato S, Ebisawa M. [Methods of Ingestion in Oral Food Challenge Tests Using Whole-Egg Powder]. Arerugi. 2016;65(3):193-199.

Table 1. Demographic and clinical characteristics of children undergoing the oral food challenge

\begin{tabular}{lllll}
\hline & All & Hen's egg & Cow's milk & Wheat \\
\hline $\mathrm{N}$ & 4930 & 2954 & 1126 & 850 \\
Age (in months) & $31(20-52)$ & $31(20-52)$ & $35(22-59)$ & $29(19-45)$ \\
Sex (male) & $3149(64 \%)$ & $1859(63 \%)$ & $699(62 \%)$ & $591(70 \%)$ \\
Elimination reasons & & & & \\
Allergic tests & $1931(23 \%)$ & $1197(41 \%)$ & $270(24 \%)$ & $464(55 \%)$ \\
Objective allergic reactions & $2999(61 \%)$ & $1757(59 \%)$ & $856(76 \%)$ & $386(45 \%)$ \\
Current allergic Complications & & & & \\
$\quad$ Asthma & $724(15 \%)$ & $417(14 \%)$ & $207(18 \%)$ & $100(12 \%)$ \\
$\quad$ Atopic dermatitis & $1492(30 \%)$ & $848(29 \%)$ & $353(31 \%)$ & $291(34 \%)$ \\
$\quad$ Allergic rhinitis & $222(5 \%)$ & $139(5 \%)$ & $66(6 \%)$ & $17(2 \%)$ \\
Total IgE (IU/mL) & $270(101-722)$ & $256(90-728)$ & $287(110-685)$ & $332(132-768)$ \\
Food-specific IgE (kUA $/ \mathrm{L})$ & - & $14.0(6.0-32.5)$ & $6.8(2.7-15.3)$ & $12.7(4.1-32.2)$ \\
Total target dose & & & & \\
Low & $1096(22 \%)$ & $590(20 \%)$ & $291(26 \%)$ & $215(25 \%)$ \\
Medium & $2020(41 \%)$ & $1152(39 \%)$ & $488(43 \%)$ & $380(45 \%)$ \\
Full & $1814(37 \%)$ & $1212(41 \%)$ & $347(31 \%)$ & $255(30 \%)$ \\
\hline
\end{tabular}

Numerical values are represented as count (\%) or median (IQR).

Symptoms listed under "elimination reasons" correspond to the number (\%) of children with a parentreported history of allergic reactions, including anaphylaxis.

Food-specific IgE shows the level of serum IgE value against egg-white, cow's milk, or wheat.

Table 2. Comparison of oral food challenge outcomes between the skin or laryngeal cases vs. control group

\begin{tabular}{|c|c|c|c|c|c|c|c|}
\hline Outcomes & Hen's egg & Hen's egg & Hen's egg & Hen's egg & Hen's egg & Cow's milk & Cow's 1 \\
\hline & Cases & & Control & $p$-value & $p$-value & Cases & \\
\hline
\end{tabular}




\begin{tabular}{llllllll}
\hline Outcomes & Hen's egg & Hen's egg & Hen's egg & Hen's egg & Hen's egg & Cow's milk & Cow's n \\
\hline \multirow{3}{*}{ Number } & Skin & Laryngeal & & Skin vs. control & Laryngeal vs. control & Skin & Laryng \\
Positive results & 114 & 25 & 2815 & & & 91 & 24 \\
Anaphylaxis & $57(67 \%)$ & $16(64 \%)$ & $647(23 \%)$ & $<0.001$ & $<0.001$ & $55(60 \%)$ & $11(45 \%$ \\
Adrenalin injection & $5(4 \%)$ & $2(8 \%)$ & $55(2 \%)$ & 0.07 & 0.03 & $8(9 \%)$ & $2(8 \%)$ \\
\hline
\end{tabular}

$p$-values were calculated using the Mann-Whitney $U$ or chi-square test. Children with mild skin signs or laryngeal symptoms emerging within 5 minutes of OFC initiation are assigned to the skin or laryngeal groups, respectively.

Food-specific $\operatorname{IgE}(\mathrm{kU} / \mathrm{L})$ is the level of $\operatorname{IgE}$ specific to either egg-white or cow's milk.

Table 3. Risk for a positive oral food challenge among children with early skin signs

\begin{tabular}{lllllll}
\hline & Hen's egg & Hen's egg & Hen's egg & Hen's egg & Cow's milk & Cow's \\
\hline Characteristics & ORs & $p$-value & aORs & $p$-value & ORs & $p$-value \\
Skin cases & $7.0(4.6-10.4)$ & $<0.001$ & $5.1(3.8-7.0)$ & $<0.001$ & $3.9(2.5-6.1)$ & $<0.001$ \\
Age (months) & $1.0(0.99-1.0)$ & 0.51 & $1.0(0.9-1.01)$ & 0.08 & $0.9(0.9-1.0)$ & 0.09 \\
Sex (male) & $1.1(0.9-1.4)$ & 0.12 & $1.2(1.0-1.3)$ & 0.04 & $0.9(0.8-1.3)$ & 0.90 \\
OFC total target dose & & & & & & \\
Low & Reference category & Reference category & & & & \\
Medium & $0.7(0.6-0.9)$ & 0.003 & $0.9(0.8-1.1)$ & 0.23 & $0.5(0.4-0.7)$ & $<0.001$ \\
Full & $0.4(0.3-0.4)$ & $<0.001$ & $0.4(0.4-0.5)$ & $<0.001$ & $0.3(0.2-0.4)$ & $<0.001$ \\
Elimination reasons & & & & & & \\
Allergic tests & Reference category & Reference category & & & & \\
Objective allergic reactions & $0.9(0.8-1.1)$ & 0.44 & $0.9(0.8-1.1)$ & 0.38 & $1.1(0.8-1.4)$ & 0.70 \\
Asthma & $0.9(0.7-1.2)$ & 0.49 & $1.0(0.8-1.2)$ & 0.77 & $1.1(0.8-1.5)$ & 0.58 \\
Atopic dermatitis & $1.2(1.0-1.5)$ & 0.04 & $1.0(0.9-1.2)$ & 0.77 & $0.9(0.7-1.2)$ & 0.50 \\
Allergic rhinitis & $0.9(0.6-1.5)$ & 0.94 & $0.9(0.6-1.3)$ & 0.43 & $0.7(0.4-1.2)$ & 0.16 \\
Total IgE (IU/mL) & $1.0(0.9-1.1)$ & 0.19 & $0.8(0.8-0.9)$ & $<0.001$ & $1.1(1.0-1.3)$ & 0.01 \\
Food-specific IgE $\left(\mathrm{kU} \mathrm{A}_{\mathrm{A}} / \mathrm{L}\right)$ & $1.4(1.3-1.5)$ & $<0.001$ & $1.5(1.4-1.7)$ & $<0.001$ & $2.0(1.7-2.2)$ & $<0.001$ \\
\hline & & & & & &
\end{tabular}

Univariate and multivariate logistic regression was used. OR, odds ratios; aOR, adjusted odds ratios. The following variables were included in the multivariate analysis: age, sex, elimination reasons, asthma, atopic dermatitis, allergic rhinitis, and serum levels of total $\operatorname{IgE}$ and food-specific IgE. Total and specific $\operatorname{IgE}$ raw values were $\log$-transformed $(\log (\mathrm{x}+1))$ prior to the analyses.

Table 4. Risk for a positive oral food challenge among children with early laryngeal symptoms

\begin{tabular}{lllllll}
\hline & Hen's egg & & & & Cow's milk \\
\hline & ORs & $p$-value & aORs & $p$-value & ORs & $p$-value \\
Laryngeal cases & $6.0(2.6-13.7)$ & $<0.001$ & $5.2(2.2-12.2)$ & $<0.001$ & $2.2(0.9-5.0)$ & 0.06 \\
Age (months) & $1.0(0.99-1.0)$ & 0.99 & $1.0(1.0-1.01)$ & 0.009 & $1.0(0.99-1.0)$ & 0.29 \\
Sex (male) & $1.2(0.9-1.4)$ & 0.12 & $1.2(0.9-1.5)$ & 0.06 & $1.1(0.8-1.4)$ & 0.73 \\
OFC total target dose & & & & & & \\
Low & Reference category & Reference category & & & & \\
Medium & $0.7(0.6-0.9)$ & 0.005 & $0.9(0.7-1.1)$ & 0.29 & $0.5(0.4-0.7)$ & $<0.001$ \\
Full & $0.4(0.3-0.4)$ & $<0.001$ & $0.5(0.4-0.6)$ & $<0.001$ & $0.3(0.2-0.4)$ & $<0.001$ \\
Elimination reasons & & & & & &
\end{tabular}




\begin{tabular}{lllllll}
\hline & Hen's egg & & & & Cow's milk \\
\hline Allergic tests & Reference category & Reference category & & & & \\
Objective allergic reactions & $0.9(0.8-1.1)$ & 0.39 & $0.9(0.8-1.1)$ & 0.53 & $1.1(0.8-1.5)$ & 0.74 \\
Asthma & $0.9(0.7-1.1)$ & 0.33 & $0.8(0.6-1.1)$ & 0.11 & $1.1(0.8-1.6)$ & 0.44 \\
Atopic dermatitis & $1.2(1.0-1.5)$ & 0.02 & $1.1(0.9-1.4)$ & 0.13 & $0.9(0.7-1.2)$ & 0.50 \\
Allergic rhinitis & $0.9(0.6-1.4)$ & 0.79 & $0.9(0.6-1.5)$ & 0.90 & $0.8(0.4-1.5)$ & 0.46 \\
Total IgE (IU/mL) & $1.0(0.9-1.1)$ & 0.14 & $0.8(0.7-0.9)$ & $<0.001$ & $1.1(1.0-1.3)$ & 0.01 \\
Food-specific IgE $(\mathrm{kU}$ A $/ \mathrm{L})$ & $1.4(1.3-1.5)$ & $<0.001$ & $1.5(1.3-1.7)$ & $<0.001$ & $1.9(1.7-2.2)$ & $<0.001$ \\
\hline
\end{tabular}

Univariate and multivariate logistic regression was used. OR, odds ratios; aOR, adjusted odds ratios. The following variables were included in the multivariate analysis: age, sex, elimination reasons, asthma, atopic dermatitis, allergic rhinitis, and serum levels of total $\operatorname{IgE}$ and food-specific IgE. Total and specific $\operatorname{IgE}$ raw values were $\log$-transformed $(\log (\mathrm{x}+1))$ prior to the analyses.

\section{Figure legends}

Figure 1. Flowchart of the procedure for the inclusion of subjects as skin or laryngeal cases and controls.

Figure 2. Receiver operating characteristics curves of the prediction models for a positive oral food challenge (OFC) result. a) Early skin signs; b) Early laryngeal symptoms. The predictive models were constructed using multivariate logistic regression and included early skin signs or laryngeal symptoms and the following predictors: age, sex, and serum levels of total immunoglobulin E (IgE) and food-specific IgE (egg white or cow's milk).

\section{Hosted file}

Figure_1.pdf available at https://authorea.com/users/392373/articles/506284-evaluation-ofearly-skin-or-laryngeal-reaction-at-oral-food-challenge

\section{Hosted file}

Figure_2.pdf available at https://authorea.com/users/392373/articles/506284-evaluation-ofearly-skin-or-laryngeal-reaction-at-oral-food-challenge

\section{Hosted file}

PAI_SI.pdf available at https://authorea.com/users/392373/articles/506284-evaluation-ofearly-skin-or-laryngeal-reaction-at-oral-food-challenge 